\title{
Non-Celiac Wheat Sensitivity as an Allergic Condition: Personal Experience and Narrative Review
}

Antonio Carroccio, $\mathrm{MD}^{1}$, Pasquale Mansueto, $\mathrm{MD}^{2}$, Alberto D’Alcamo, $\mathrm{MD}^{2}$ and Giuseppe Iacono, $\mathrm{MD}^{3}$

OBJECTIVES: Non-celiac wheat sensitivity (NCWS) is a newly described clinical entity characterized by symptoms, which can involve the gastrointestinal tract, the nervous system, the skin, and other organs. There is little data on the pathogenesis of NCWS and it is probable that different pathogenic mechanisms are involved in the different clinical manifestations of the disease. The only common denominator of NCWS "syndrome" is wheat consumption: the symptoms disappear on exclusion of wheat from the diet, and reappear on wheat consumption. The objective of this study was to review our prior data regarding NCWS and to review relevant medical literature regarding NCWS, with particular attention to the hypothesis that NCWS patients could suffer from non-immunoglobulin E (IgE)-mediated wheat allergy.

METHODS: $\quad$ We reviewed our data on 276 patients diagnosed with NCWS by means of double-blind placebocontrolled (DBPC) wheat challenge. The data indicating a possible wheat allergy diagnosis were examined and other data in the literature were reviewed; we review the role of serum immunoglobulin $\mathrm{G}$ antibodies and the basophil activation assay in food allergy, and the histology findings in the food allergy diagnosis.

RESULTS: $\quad$ The comparison between patients suffering from NCWS and presenting with irritable bowel syndrome (IBS) and controls with IBS not due to NCWS showed that NCWS was characterized by: a personal history of food allergy in the pediatric age $(0.01)$, coexistent atopic diseases $(0.0001)$, positive serum anti-gliadin $(0.0001)$ and anti-betalactoglobulin $(0.001)$ antibodies, positive cytofluorimetric assay revealing in vitro basophil activation by food antigens $(0.0001)$, and a presence of eosinophils in the intestinal mucosa biopsies (0.0001).

CONCLUSIONS: Patients with NCWS and multiple food sensitivity show several clinical, laboratory, and histological characteristics that suggest they might be suffering from non-IgE-mediated food allergy. However, other pathogenic mechanisms need to be considered.

Am J Gastroenterol 2013; 108:1845-1852; doi:10.1038/ajg.2013.353; published online 5 November 2013

\section{INTRODUCTION}

The definition of NCWS

In the last 5 years since the publication of the very stimulating article by Elena Verdù about patients with irritable bowel syndrome-like (IBS-like) symptoms who fully resolve their symptoms on gluten-free diet even though they do not fulfill the diagnostic criteria for celiac disease (CD) or wheat allergy (1), there has been scientific interest in a possibly new clinical condition, which has been called a "fertile crescent for research" (2). This condition was initially named "gluten sensitivity" $(3,4)$, but subsequently the term "non-celiac gluten sensitivity"

(5) was preferred to better distinguish it from CD. However, it is not known which components of wheat cause the symptoms in non-celiac gluten sensitivity patients, and because there is no definite proof that gluten is really the culprit (6), we prefer the term "non-celiac wheat sensitivity" (NCWS) (7).

\section{The spectrum of NCWS symptoms}

Many gastrointestinal and extra-gastrointestinal symptoms have been attributed to NCWS. Table 1 summarizes the clinical symptoms reported in the literature and those which we have observed in our patients $(4,7-10)$. Symptoms can affect both gastrointestinal and extra-gastrointestinal systems and it is probable that this list will change in the next few years, including new manifestations,

${ }^{1}$ Internal Medicine, Hospital of Sciacca, ASP Agrigento and University of Palermo, Palermo, Italy; ${ }^{2}$ Internal Medicine, University of Palermo, Palermo, Italy; ${ }^{3}$ Pediatric Gastroenterology, “Di Cristina” Children's Hospital, Palermo, Italy. Correspondence: Antonio Carroccio, MD, Internal Medicine, University of Palermo, via Ciaculli 207, Palermo 90124, Italy. E-mail: acarroccio@hotmail.com

Received 21 February 2013; accepted 3 September 2013 


\begin{tabular}{|c|c|c|}
\hline $\begin{array}{l}\text { Gastrointestinal } \\
\text { symptoms }\end{array}$ & $\begin{array}{l}\text { Neurological-psychiatric } \\
\text { symptoms }\end{array}$ & Other symptoms \\
\hline Abdominal pain & Foggy mind & Eczema \\
\hline Bloating & Tiredness & Skin rash \\
\hline Diarrhea & Headache & Joint pain \\
\hline Constipation & Depression & Muscle pain \\
\hline $\begin{array}{l}\text { Alternating bowel } \\
\text { movements }\end{array}$ & $\begin{array}{l}\text { Numbness in arms, legs, } \\
\text { and fingers }\end{array}$ & Oligo- or poly-menorrhea \\
\hline Nausea & Hyposthenia & Anemia \\
\hline Vomiting & Loss of balance & Weight loss \\
\hline Hematochezia & Disturbed sleep pattern & Weight increase \\
\hline \multirow[t]{3}{*}{ Anal fissures } & Mood swings & Puffiness \\
\hline & & Interstitial cystitis \\
\hline & & Ingrown hairs \\
\hline
\end{tabular}

but also excluding some of those now listed. For example, although there is general agreement that patients suffering from NCWS can have neurological manifestations, it is unknown whether some psychiatric symptoms (i.e., depression) can be considered a direct consequence of wheat ingestion or are dependent on a patient's poor quality of life. Similarly, some patients have reported weight loss but it is not clear whether this is related to a malabsorption syndrome or self-limited diet because of fear of symptoms after meals. On the contrary, some patients report weight gain on a wheat-containing diet and a dramatic weight normalization after wheat exclusion from their diet. What is counterintuitive is that the patients subjectively report that their daily caloric intake remains identical or even higher. In these cases also, an accurate evaluation of the glycemic index of the foods consumed before and after the wheat-free diet should be mandatory, as a simple modification in the insulin levels could be the basis of the body weight change. In general, it should be emphasized that such a large and heterogeneous list of manifestations very probably cannot have a single pathogenic mechanism. In this context, wheat ingestion could cause different symptoms by different mechanisms so NCWS cannot be considered a distinct clinical condition. In other words, NCWS cannot be considered "the sister" of CD; that is not the proper model, according to our analysis.

To minimize possible errors in the study of the NCWS, the study population needs to be specifically defined and carefully grouped according to the clinical presentation. That was done in patients with IBS diagnosed according to the Rome II criteria (7). The results of that study and our previous clinical experience suggested to us that a portion of NCWS patients with IBS could be suffering from non-immunoglobulin E (IgE)-mediated food hypersensitivity $(\mathrm{FH})$. The aims of this study are: (a) to re-evaluate the results of the double-blind placebo-controlled (DBPC) food challenges in patients with IBS, underlining the results which could suggest an allergic pathogenesis; (b) perform a narrative review of the use of DBPC challenge, the role of serum immunoglobulin G (IgG) antibodies, and the basophil activation assay in food allergy, and the histology findings in the food allergy diagnosis.

\section{METHODS}

All patients included in the previous study were prescribed a standard elimination diet with the exclusion of wheat, cow's milk (CM), eggs, tomato, and chocolate (7). Patients self-reporting FH were asked to avoid ingestion and/or contact with the food(s) causing symptoms. After 4 weeks on elimination diet, they underwent DBPC challenges. We performed the wheat challenge by administering a daily dose $13 \mathrm{~g}$ of flour, equivalent to about $20 \mathrm{~g}$ of bread. A total of 12 capsules daily were given, three times daily, away from meals. DBPC challenge for CM proteins was performed with an identical method, administering capsules containing casein, lactoalbumin, and lactoglobulin, at least 4 weeks before or after wheat challenge and when the patients were asymptomatic, on elimination diet. Xylose was used as placebo. The shape and size of the capsules were identical in each case. Further details have been previously published (7). In this study, we re-evaluated the results of the DBPC challenges in the IBS patients included in a previous study (7). The Fisher's test was used for frequency comparison; a $P$ value $<0.05$ was considered statistically significant.

\section{RESULTS}

\section{The DBPC wheat challenge in patients with IBS-like manifestations}

About one-third of $>900$ patients with IBS resolved their symptoms on elimination diet and relapsed into symptoms on DBPC wheat challenge (7). Table 2 summarizes the clinical and laboratory characteristics of the patients with NCWS and multiple food sensitivity included in that study (7), which can suggest an allergic background; for comparison, patients with IBS not due to food sensitivity (i.e., not improved on elimination diet) were selected. A personal history of food allergy in the pediatric age, coexistent atopic diseases, positive serum anti-gliadin and anti-betalactoglobulin antibodies, positive cytofluorimetric assay revealing in vitro basophil activation by food antigens, and presence of eosinophils in the intestinal mucosa biopsies, seem strong indicators of FH. Although demonstrating the existence of NCWS by means of the DBPC challenge method is probably the main result of that study, we believe it is of great interest to have revealed that 206 of the 276 patients suffering from NCWS showed the same IBS-like symptoms on CM protein DBPC challenge. Furthermore, the majority of those same patients showed symptoms, on open challenge, with egg, chocolate, and other foods.

\section{DISCUSSION AND NARRATIVE REVIEW OF THE LITERATURE}

The evidence that the majority of NCWS patients included in our study also exhibited CM protein hypersensitivity (7), leads 
us to suggest that a great percentage of NCWS could be categorized as being affected by "multiple FH," a condition, which is well known in the pediatric literature $(11,12)$. In this regard, the fundamental role of the DBPC method must be stressed in the diagnosis of FH in patients with gastrointestinal manifestations. Definite proof of underlying immunological mechanisms is lacking for most gastrointestinal disorders due to food allergy, except for those mediated by food-specific IgE antibodies (13). However, because most gastrointestinal food allergies are not mediated by IgE antibodies, alternative assays in the determination of eosinophil by-products in biopsy specimens or fecal samples have been investigated; no such tests with acceptable diagnostic accuracy have been found (13).

The same considerations should apply to the diagnosis of NCWS. It has been clearly demonstrated that wheat can cause gastrointestinal symptoms in the context of FH and multiple FH. For example, utilizing the DBPC challenge method, we found that a subgroup of children with chronic constipation due to CM allergy (14), which did not resolve on CM-free diet, were suffering from wheat hypersensitivity also, and that in these patients the exclusion of wheat, egg, and other foods resolved the symptom (15). Similar data subsequently reported that in adult patients with chronic constipation unresponsive to laxative treatment, CM-free and wheat-free diet were effective in treating the symptoms (16).

The hypothesis that in a percentage of cases NCWS is a manifestation of non-IgE-mediated food allergy, is strengthened by the higher frequency of a history of food allergy in the pediatric age that we found in our cases (see Table 2). Although we used the Fisher's test instead of a multiple analysis and this could have determined a type 1 error, the high frequency of history of food allergy in the pediatric age in the adults suffering from NCWS is an important datum, which we have observed in daily clinical practice. In this respect, Table 3 summarizes some questions that we regularly pose to adult patients with suspected NCWS or FH (i.e., self-reported wheat-intolerant patients). We are now evaluating the diagnostic accuracy of this questionnaire to propose a possible score. In any case, it is surely useful in daily practice to decide which patients have a higher probability of suffering from NCWS and should be placed on an elimination diet, and whoever has a lower probability and should be directed to a "conventional" treatment for IBS.

Obviously, the response to the elimination diet and to the subsequent challenge justifies or excludes a diagnosis of NCWS or multiple FH.

\section{A diagnostic role for serum IgG in NCWS}

Our data and the data of others showed that about $50-60 \%$ of NCWS patients had elevated serum values of IgG anti-gliadin antibodies (AGAs) $(7,8)$. Although the use of these antibody assays in CD diagnosis is advised against (17), according to this finding we think that when faced with a patient who has symptoms because of the consumption of gluten-containing foods, it is logical to test both anti-transglutaminase assay-the serum gold standard in reaching CD diagnosis-and anti-gliadin assay. This
Table 2. Clinical and laboratory data that could suggest a diagnosis of MFH in patients with NCWS and CMPI demonstrated by DBPC challenges

\begin{tabular}{|c|c|c|c|}
\hline & $\begin{array}{c}\text { NCWS + CMPI } \\
(n=206)\end{array}$ & $\begin{array}{l}\text { IBS controls } \\
(n=50)\end{array}$ & $P$ value \\
\hline $\begin{array}{l}\text { History of food allergy in } \\
\text { infancy }\end{array}$ & 40/206 (19\%) & $2 / 50(4 \%)$ & 0.01 \\
\hline Coexistent atopic disease & 73/206 (35\%) & $3 / 50(6 \%)$ & 0.0001 \\
\hline $\begin{array}{l}\text { Positive serum anti-gliadin } \\
\text { IgG }\end{array}$ & $134 / 206(65 \%)$ & $7 / 50(14 \%)$ & 0.0001 \\
\hline $\begin{array}{l}\text { Positive serum } \\
\text { anti-betalactoglobulin IgG }\end{array}$ & 80/206 (39\%) & $7 / 50(14 \%)$ & 0.001 \\
\hline $\begin{array}{l}\text { In vitro basophil activation } \\
\text { assay }\end{array}$ & $166 / 206(80 \%)$ & $2 / 50(4 \%)$ & 0.0001 \\
\hline $\begin{array}{l}\text { Intraepithelial eosinophil } \\
\text { infiltration in the colon } \\
\text { mucosa }\end{array}$ & $154 / 206(75 \%)$ & $0 / 35$ & 0.0001 \\
\hline \multicolumn{4}{|c|}{$\begin{array}{l}\text { CMPI, cow's milk protein intolerance; DBPC, double-blind placebo-controlled; } \\
\text { HPF high-power field; IBS, irritable bowel syndrome; IgG, immunoglobulin G; } \\
\text { MFH, multiple food hypersensitivity; NCWS, non-celiac wheat sensitivity. } \\
\text { As controls we included a group of patients with IBS not due to food intoler- } \\
\text { ance (from reference Carroccio et al. (7)). }\end{array}$} \\
\hline \multicolumn{4}{|c|}{$\begin{array}{l}\text { The Fisher's test was used for frequency comparison; a } P \text { value }<0.05 \text { was } \\
\text { considered statistically significant. Eosinophils were counted at a HPF }(40 x) \text {; } \\
\text { the presence of }>4 \text { eosinophils per HPF in the colon mucosa biopsy was } \\
\text { considered "positive". }\end{array}$} \\
\hline
\end{tabular}

choice is precisely because the AGA assay may help confirm a suspicion of NCWS. Furthermore, it is of interest that Ruuskanen et al. (18) showed that HLA-DQ2-positive or HLA-DQ8-positive subjects who were tested positive for serum AGA had more severe gastrointestinal symptoms than the antibody-negative subjects. However, it must be emphasized that the positive predictive value of the AGA assay in NCWS diagnosis is very low, as it is also positive in $10-14 \%$ of patients with IBS who do not improve on elimination diet (7).

In our hypothesis, the high levels of serum AGA, as the high levels of IgG anti-betalactoglobulin antibodies, could be an indicator of FH, but it must be emphasized that the data on this are very controversial. Burks et al. (19) examined antibody responses to milk proteins in patients with milk protein intolerance shown by oral challenge, and found no increase in IgG antibodies after positive challenge. Shek et al. (20) concluded that food-specific IgG or IgG4 do not add any information to the food allergy diagnostic workup, that is, milk-induced IgE-mediated and nonIgE-mediated disorders. In contrast, the literature suggests that food-specific IgG presence is a marker of food exposure and tolerance, as seen in those participating in oral immunotherapy studies (21).

Based on the above-mentioned studies, it has been affirmed that the inappropriate use of food-specific IgG assay might only increase the likelihood of false diagnoses, resulting in unnecessary dietary restrictions and decreased quality of life (22). As a result of these serious and growing concerns, the Canadian Society of 
Table 3. Questions which, in our experience, can be useful to pose to the patients with suspected NCWS or MFH

Questions
Did you suffer from food allergy in
infancy?
If yes: how was the diagnosis was
made:
DBPC challenge? Open challenge?
IgE-based assays?

If you don't know whether you suffered from food allergy or not: did you change the kind of milk in infancy? Did you have a pediatric history of "milk intolerance"? Did you have problems at the time of weaning?

In patients with IBS-constipation: have you suffered from chronic constipation since infancy and the pediatric age?

Have you suffered (or are suffering) from allergic rhinitis or conjunctivitis or bronchial asthma or atopic dermatitis?

Comments

A history of food allergy in infancy is more frequent in NCWS patients than in IBS patients.

If yes: although quite vague, these elements can suggest a possible condition of cow's milk protein hypersensitivity in infancy or of wheat/multiple food hypersensitivity.

Did you suffer from umbilical erythema (presence of erythematous skin in the umbilical and periumbilical region, with or without secretion)

in the pediatric age, or are you suffering from it now?

Did you suffer from perianal erythema (presence of erythematous skin in the anal and perianal region) in the pediatric age, or are you suffering from it now?

DBPC, double-blind placebo-controlled; IBS, irritable bowel syndrome; MFH, multiple food hypersensitivity; NCWS, non-celiac wheat sensitivity. A positive response increases the probability of a correct diagnosis of NCWS or MFH.

Allergy and Clinical Immunology (22), the American Academy of Allergy Asthma and Immunology (23), and the European Academy of Allergy and Clinical Immunology (24) have warned against the inappropriate measurement of food-specific IgG or IgG4 to suggest the presence of potential adverse food reactions. Recent guidelines emphasize that such testing has no role in the diagnosis of food allergy or intolerance (25).

In strong opposition to the above mentioned studies and position papers, Zar et al. $(26,27)$ demonstrated that food-specific IgG4 antibody-guided exclusion diet improves symptoms in IBS patients and is associated with rectal compliance improvement. Atkinson et al. (28) used a double blind, randomized, controlled, parallel design in which IBS patients were randomized to either a "true" diet or a "sham" diet control group, based on the presence/ absence of food-specific IgG antibodies to a panel of 29 different food antigens, which showed that food elimination based on IgG antibodies may be effective in reducing IBS symptoms. Our group also showed a very high diagnostic accuracy of IgG anti-betalactoglobulin assay in pediatric patients with IBS-like (29) or gastroesophageal reflux disease (30).

The assay of IgG for food antigens in the suspicion of multiple $\mathrm{FH}$, including NCWS, should be discouraged and the elimination diet based on the results of these assays should not be advised. However, until a reliable serum marker of NCWS is found, serum IgG AGA could be helpful.

The usefulness of basophil activation assay in NCWS diagnosis The basophil activation assay, or basophil activation test (BAT), is an in vitro assessment of allergic response that requires only a small quantity of whole blood and allows measurement of a functional response, beyond just the presence of IgE class antibody. The assay currently uses flow cytometry to detect upregulation, after antigen stimulation, of some activation markers (e.g., CD63 or CD203c) usually expressed on the cell surface, in order to identify activated basophils (31).

BAT has been shown to be sensitive and specific for the diagnosis of IgE-mediated hypersensitivity reactions to pollens, hymenoptera venom, natural rubber latex allergy, and drugs (32-34). In regard to food allergy, it has been demonstrated that BAT could be more specific than skin PRICK test and/or food-specific IgE (35), and it is a reliable tool for the diagnosis of CM allergy in pediatric patients, with sensitivity similar to routine diagnostic tests but with higher specificity (36).

However, there have been very few studies on the possible usefulness of BAT in patients with IBS-like symptoms and suspected NCWS or food-hypersensitivity. In this setting, we have previously shown conflicting data. In a prospective study, we evaluated the efficacy of BAT in the diagnosis of multiple FH in IBS-like patients (37). In this study, BAT diagnosed NCWS with $86 \%$ sensitivity, $88 \%$ specificity, and $87 \%$ accuracy. This diagnostic accuracy can be considered excellent in a field that has no serologic marker to aid in the diagnosis. However, the commercial assay that we had used in the past changed methods, and since the year 2010 a new assay on whole blood replaced the old assay performed on separated leukocytes. Consequently, in our daily clinical practice we observed a fall in the accuracy of the assay; for this reason in a subsequent study we compared the "old" and the "new" BAT methods (38). Our results showed that the new BAT CD63-based detection on whole blood samples is not useful in FH and NCWS diagnosis. Furthermore, data from Ciacci et al. do not confirm the usefulness of BAT in NCWS diagnosis (39).

In summary, BAT accuracy in NCWS diagnosis seems excessively influenced by a method that is not standardized and reproducible. The use of a commercial kit cannot be recommended, but in each center the results of a "home-made assay" could be compared with the results of DBPC wheat challenge to obtain an internal reference of BAT accuracy. Whatever is the real percentage of positive BAT in NCWS patients, the evidence of basophil cell activation after wheat in vitro challenge adds strength to the hypothesis of an allergic pathogenesis in a subgroup of NCWS. 


\section{The association between NCWS and atopic diseases}

In regard to a possible association between NCWS and atopic diseases, Massari et al. (40) found a very high frequency (29\%) of duodenal mucosa lesions in patients with atopic diseases (asthma, rhinitis, or contact dermatitis); all patients were negative for CD-specific serum antibodies (endomysial antibodies) and showed gastrointestinal problems, which disappeared on gluten-free diet and the authors concluded that NCWS commonly occurs in allergic patients. Although some limitations of the study must be noted, that is, patients with intestinal villi atrophy were included-the frequency of the association reported by the authors is surprising.

The other side of this relationship was shown in our recent study (7). We found that 73 of 206 patients suffering from NCWS associated with CM protein hypersensitivity showed coexistent atopic diseases: a frequency significantly higher than that observed in patients suffering from IBS. In general, the relationship between $\mathrm{FH}$ and atopic manifestations, although investigated in patient-based studies, has been scarcely evaluated in the general population.

According to a recent study of the Centers for Disease Control and Prevention, children with food allergy are about 2-4 times more susceptible to develop further clinical manifestations, such as respiratory allergies (3.6-fold), asthma (4.0-fold), and atopic dermatitis (2.4-fold), compared with children without food allergy (41). Several studies reported the co-occurrence of other allergic conditions in adult patients with food allergy, such as: asthma (34-49\%), allergic rhinitis (33-40\%), and atopic dermatitis $(35-71 \%)(42,43)$. However, it must be said that the above studies (41-43) referred to patients with IgE-mediated food allergy, a condition that-by definition-excludes NCWS. In general, the preliminary data that seem to indicate a higher frequency of atopic diseases in NCWS patients and NCWS in atopic patients support the hypothesis that a subgroup of NCWS patients suffer from non-IgE-mediated food allergy.

\section{Histology in NCWS: elements that can support the food allergy hypothesis}

Duodenal histology in NCWS patients has been evaluated. Villous atrophy has never been reported, although it is a tautological finding because in the presence of villous atrophy NCWS diagnosis is excluded by definition (4). In our experience, there are very few patients who could be included in the diagnostic category of "NCWS plus CM protein hypersensitivity" and who showed mild-to-moderate duodenal villous atrophy (unpublished data). Obviously, we are referring to patients who did not display DQ2 and DQ8 alleles. Owing to the need to explore NCWS while avoiding further confounding elements, we agree that the doubtful cases, that is, those showing intestinal villi damage, must be excluded.

Consequently, the duodenal histology of NCWS is reported as normal (Marsh 0) or characterized by an infiltration of intraepithelial lymphocytes (IELs; Marsh 1), in absence of villi atrophy. Sapone et al. (44) reported $>30$ IEL/100 enterocytes in 22 of 26 "gluten-sensitive patients", and Volta et al. (8) found
Marsh 1 histology in 33 of 78 (42\%) cases. Our recent retrospective study (7) showed a duodenal mucosa IEL increase ( $>25$ IEL/enterocytes) in 248 of 276 cases (90\%). In our study (7), as in that of Sapone et al. (44), the entity of IEL infiltration was intermediate between those observed in patients with CD and in controls with "functional" gastrointestinal disorders (dyspepsia or IBS). However, IEL infiltration of the duodenal mucosa can be due to several causes (45), so it is not possible to consider this finding specific of wheat sensitivity.

A more plausible histology marker of NCWS suggested by our experience is the mucosal eosinophil infiltrate. Colon histology evaluation showed intraepithelial and lamina propria infiltration of eosinophils in about two-thirds of cases and this finding was also frequently observed in the duodenum (7). We found $>4$ intraepithelial eosinophils (at a high-power field: $40 \times$ ) in the colon mucosa in 174 of 276 (63\%) NCWS patients; furthermore, the count of the duodenal intraepithelial eosinophils was significantly higher in NCWS patients than in IBS controls (7). In an experimental model, it has been shown that oral antigen can induce eosinophilic inflammation in various segments of the gastrointestinal tract, including the esophagus, stomach, small intestine, and Peyer's patches (46). It is known that both the mixed IgE-mediated and non-IgE-mediated diseases comprise distinct hypersensitivity disorders that are characterized by a pronounced eosinophil infiltration of the gastrointestinal tract and have been referred to as eosinophil-associated gastrointestinal disorders (47).

In several previous studies, we found that the eosinophil infiltrate in the intestinal mucosa characterized the patients suffering from chronic constipation due to food allergy $(12,13)$. Similar histological data were reported in school-age children with rectal bleeding due to allergic procto-colitis (48). It is also interesting that in 70 of 206 (35\%) patients with NCWS and CM protein hypersensitivity, we found the presence of lymphoid hyperplasia in the colon mucosa (7). This histology characteristic has been reported in adult patients (49) and children (50) with rectal bleeding due to food allergy. In clinical practice, the eosinophil infiltrate and/or the presence of lymphoid hyperplasia in the colon or duodenal mucosa cannot be considered an accurate diagnostic marker of NCWS. However, their presence could recommend an elimination diet; the clinical response to the diet and the subsequent DBPC challenge confirm the diagnosis.

\section{Alternative and supplementary hypotheses}

Although we have shown several aspects that could indicate an allergic condition at the basis of the functional bowel disease in NCWS patients, it is very probable that different pathogenic mechanisms are responsible for IBS-like symptoms in different subgroups of patients or even in the same patient.

First, some proteins in wheat are difficult to digest as the human intestine physiologically lacks the enzymes, which should cut the protein chains into single amino acids. Residual long peptides could have a role in causing functional digestive problems. Furthermore, it is becoming more difficult to 
exclude a CD diagnosis in patients who self-report symptoms related to wheat ingestion. The presence of duodenal villi atrophy cannot be considered mandatory for CD diagnosis, although it is still included in the current diagnostic criteria for adult patients. It has been demonstrated that serum anti-endomysial-positive patients with an initial evaluation of normal duodenal histology had symptom resolution on glutenfree diet, and they eventually developed duodenal villi atrophy on gluten-containing diet $(51,52)$. The objection that these CD patients tested positive for serum CD-specific antibodies (anti-transglutaminase and anti-endomysium), whereas NCWS must be negative by definition, could be disregarded considering that in a very initial phase of $\mathrm{CD}$ the specific antibodies can be detected only in the intestinal mucosa, the site of their production, and not in the serum $(53,54)$. Despite the negative serum anti-transglutaminase and anti-endomysium assays and the absence of villous atrophy, we demonstrated that symptomatic patients who produced anti-endomysium in the duodenal culture can subsequently develop villous atrophy when remaining on a gluten-containing diet (55). In our recent study, anti-endomysium production in duodenal culture was found in 22 of $276(8 \%)$ patients diagnosed with NCWS, thus not showing villi atrophy and positive serum antibodies (7). It is possible that future diagnostic criteria for $\mathrm{CD}$ will include these patients.

Other pathogenic mechanisms, even if not immune-mediated, should be considered. For example, it is possible that the non-protein component of wheat might be responsible for symptoms. The fermentable fructans could cause IBS-like symptoms (6), and it has been demonstrated that fermentable oligo-, di-, and monosaccharides and polyols may cause symptoms, which overlap with IBS (56). A wheat-free diet may be useful in these patients, as it reduces the amount of the short-chain carbohydrates present in processed grains and cereals (57). This hypothesis was recently further supported by a study, which demonstrated that in self-reported NCWS patients the gastrointestinal symptoms significantly improved during reduced fermentable oligo-, di-, and mono-saccharides and polyols intake, but significantly worsened to a similar degree when their diets included gluten or whey protein. This study did not show glutenspecific gastrointestinal effects (58). However, it should be noted that the patients included in that study were not representative of NCWS as all subjects with duodenal lymphocitosis (Marsh 1) were excluded and most of the NCWS patients included in other studies showed a Marsh 1 histology. Also the use of whey proteins as placebo could be confounding as we had demonstrated a very high frequency of NCWS associated with CM protein hypersensitivity (7).

A change in diet with wheat withdrawal can also cause a change in the intestinal microbiota. This is now considered a crucial element in IBS pathogenesis (59). Future studies in NCWS patients should also consider the role of diet in the microbiota and, in turn, on the intestinal immune system. In an animal model, Verdù et al. (60) showed that mice sensitized to gliadin displayed intrinsic muscle hypercontractility associated with increased release of acetylcholine from the myenteric plexus. This finding was associated with HLA-DQ8-bound gluten peptides; HLA-DQ6 controls did not exhibit the abnormalities in gut function observed in DQ8 mice. The authors concluded that gluten sensitivity in HLA-DQ8 mice can induce immune activation in the absence of intestinal atrophy. It could be of interest to connect the above results with our recent data showing an elevated anal sphincter pressure due to wheat and CM-hypersensitivity in patients with anal fissures (61). In these patients, a wheat-free diet led to a reduction in anal sphincter pressure and a high rate of anal fissure healing.

Recently, there was further evidence that HLA-DQ2/DQ8positive patients suffering from diarrhea-predominant IBS showed higher small bowel and colon permeability on a diet containing gluten than on a diet containing rice. These patients also showed significant decreases in expression of zonula occludens 1, claudin-1, and occludin in rectosigmoid mucosa on the glutencontaining diet. On the whole, these data confirmed that gluten alters the bowel barrier functions in patients with IBS-diarrhea, particularly in HLA-DQ2/DQ8-positive patients (62).

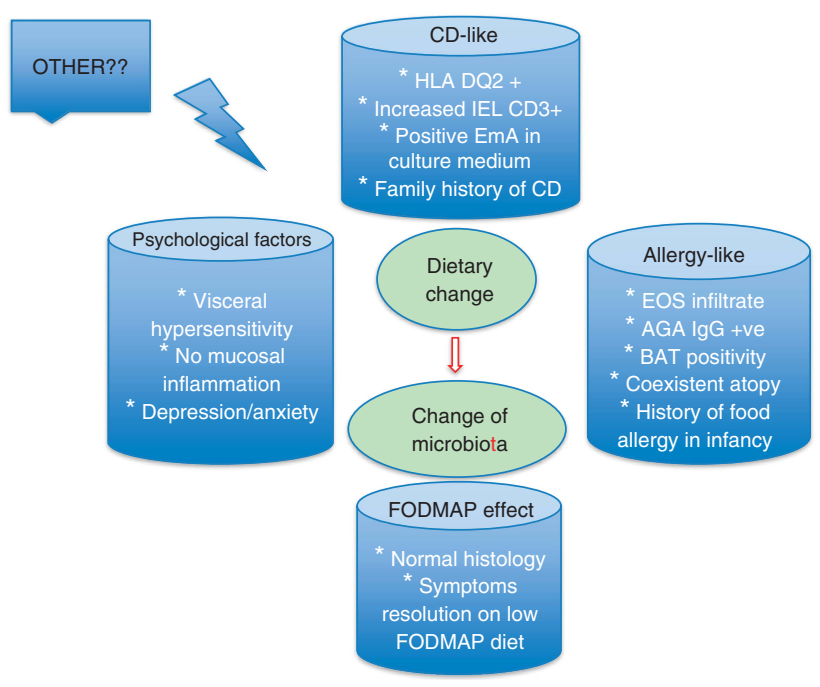

Figure 1. NCWS patients. Hypotheses of different subgroups of NCWS patients, according to different pathogenic mechanisms. Patients in the upper side of the figure (CD-like) have clinical and laboratory characteristics, which resemble $C D$, although they do not fulfill the current diagnostic criteria for CD; it is probable that they can develop CD in the future. Patients on the right side of the figure (allergy-like) show clinical laboratory characteristics, which resemble food allergy; this group is, in our experience, the most numerous. Patients on the lower side of the figure show no signs of duodenal histology inflammation and benefit from a low FODMAP diet-they could not be considered as really affected by NCWS. Patients on the left side of the figure are those with "typical IBS" related to psychosomatic mechanisms: in these patients NCWS could be excluded by means of DBPC challenge. The middle of the figure underlines the possible role of the microbiota and the change that a restricted diet (i.e., gluten free) can cause in the intestinal flora. This could be a key element to understand the effect of the dietary change on intestinal symptoms. Other factors, rarely studied nowadays, should have a role in causing NCWS. AGA, anti-gliadin antibody; BAT, basophil activation test; CD, celiac disease; DBPC, double-blind placebocontrolled; EmA, endomysial antibody; EOS, eosinophils; FODMAP, fermentable oligo-, di-, and mono-saccharides and polyols; IEL, intraepithelial lymphocyte; IgG, immunoglobulin G; NCWS, non-celiac wheat sensitivity. 
In conclusion, NCWS can now be considered the cause of gastrointestinal symptoms, which overlap those commonly attributed to functional disorders. However, many doubts remain and it must be underlined that we must utilize the DBPC challenge method to confirm the suspicion of NCWS and then study the pathogenesis of that specific clinical manifestation. A confident NCWS diagnosis must exclude a placebo effect. In our opinion, NCWS is a heterogeneous condition, which includes different subgroups of patients (Figure 1). We suggest that the patients suffering from NCWS alone are probably different from those suffering from multiple food sensitivity. This latter group of patients is more numerous, including a number of cases threefold greater than the patients suffering from wheat sensitivity alone. The patients with NCWS and multiple food sensitivity show several clinical, laboratory, and histology characteristics, which suggest that they might suffer from non-IgE-mediated food allergy. Future studies on the pathogenic mechanisms of this form of NCWS will clarify whether "NCWS" can be replaced by the shorter "wheat hypersensitivity".

\section{CONFLICT OF INTEREST}

Guarantor of the article: Antonio Carroccio, MD.

Specific author contributions: Antonio Carroccio conceived and initiated the research and corrected the manuscript, Pasquale Mansueto performed PubMed research and wrote the text, Alberto D'Alcamo performed PubMed research and wrote the text, Giuseppe Iacono conceived the research and corrected the manuscript. Financial support: There are no financial or editorial assistance received to support the research project and/or preparation of the article.

\section{Potential competing interests: None.}

\section{ACKNOWLEDGMENTS}

We thank the native English speaker Frank Adamo for revising the text. We also thank Maurizio Soresi, MD, for his help in the statistical analysis and in replying to the reviewers' comments on the statistical methods.

\section{REFERENCES}

1. Verdu EF, Armstrong D, Murray JA. Between celiac disease and irritable bowel syndrome: the no man's land of gluten sensitivity. Am J Gastroenterol 2009;104:1587-94.

2. Ball AJ, Hadjivassiliou M, Sanders DS. Is gluten sensitivity a "no man's land" or a "fertile crescent" for research? Am J Gastroenterol 2010;105:222-3.

3. Troncone R, Jabri B. Celiac disease and gluten-sensitivity. J Intern Med 2011;269:582-90.

4. Sapone A, Bai J, Ciacci C et al. Spectrum of gluten-related disorders: consensus on new nomenclature and classification. BMC Med 2012;10:13.

5. Ludvigsson JF, Leffler DA, Bai JC et al. The Oslo definitions for coeliac disease and related terms. Gut 2013;62:43-52.

6. Ferch CC, Chey WD. Irritable bowel syndrome and gluten sensitivity without celiac disease: separating the wheat from the chaff. Gastroenterology 2012;142:664-6

7. Carroccio A, Mansueto P, Iacono G et al. Non-celiac wheat sensitivity diagnosed by double-blind placebo-controlled challenge: exploring a new clinical entity. Am J Gastroenterol 2012;107:1898-906.

8. Volta U, Tovoli F, Cigola R et al. Serological tests in gluten-sensitivity. J Clin Gastroenterol 2012;46:680-5.

9. Lundin KE, Alaedini A. Non-celiac gluten sensitivity. Gastrointest Endoscopy Clin N Am 2012;22:723-34.
10. Anonymous PatientRostami K, Hogg-Kollars S. Non coeliac gluten sensitivity. BMJ 2012;345:e7982.

11. Carroccio A, Cavataio F, Montalto G et al. Intolerance to hydrolysed cow's milk proteins in infants: clinical characteristics and dietary treatment. Clin Exp All 2000;30:1598-603.

12. Iacono G, Carroccio A, Cavataio F et al. Use of ass' milk in multiple food allergy. J Ped Gastroenterol Nutr 1992;14:177-81.

13. Sampson HA, Sicherer SH, Birnbaum AH. AGA technical review on the evaluation of food allergy in gastrointestinal disorders. Gastroenterology 2001;120:1026-40.

14. Iacono G, Cavataio F, Montalto G et al. Intolerance of cow's milk and chronic constipation in children. N Engl J Med 1998;338:1100-4.

15. Carroccio A, Scalici C, Maresi M et al. Chronic constipation and food intolerance: a model of proctitis causing constipation. Scand J Gastroenterol 2005;40:33-42.

16. Carroccio A, Di Prima L, Iacono G et al. Multiple food hypersensitivity as a cause of refractory chronic constipation in adults. Scand J Gastroenterol 2006;41:498-504.

17. Fasano A, Catassi C. Celiac disease. N Engl J Med 2012;367:2419-26.

18. Ruuskanen A, Luostarinen L, Collin P et al. Persistently positive gliadin antibodies without transglutaminase antibodies in the elderly: gluten intolerance beyond coeliac disease. Dig Liver Dis 2011;43:772-8.

19. Burks AW, Williams LW, Casteel HB et al. Antibody response to milk proteins in patients with milk protein intolerance documented by challenge. J Allergy Clin Immunol 1990;85:921-7.

20. Shek LP, Bardina L, Castro R et al. Humoral and cellular responses to cow milk proteins in patients with milk-induced IgE-mediated and non-IgEmediated disorders. Allergy 2005;60:912-9.

21. Skripak JM, Nash SD, Rowley H et al. A randomized, double-blind, placebo-controlled study of milk oral immunotherapy for cow's milk allergy. J Allergy Clin Immunol 2008;122:1154-60.

22. Carr S, Chan E, Lavine $\mathrm{E}$ et al. CSACI position statement on the testing of food-specific IgG. Allergy Asthma Clin Immunol 2012;8:12.

23. Bock SA. AAAAI support of the EAACI position Paper on IgG4. J Allergy Clin Immunol 2010;125:1410.

24. Stapel SO, Asero R, Ballmer-Weber BK et al. Testing for IgG4 against foods is not recommended as a diagnostic tool: EAACI Task Force Report. Allergy 2008;63:793-6.

25. Boyce JA, Assaad A, Burks AW et al. Guidelines for the diagnosis and management of food allergy in the United States: summary of the NIAIDsponsored expert panel report. J Allergy Clin Immunol 2010;126: 1105-18.

26. Zar S, Benson MJ, Kumar D. Food-specific serum IgG4 and IgE titers to common food antigens in irritable bowel syndrome. Am J Gastroenterol 2005; 100:1550-7.

27. Zar S, Mincher L, Benson MJ et al. Food-specific IgG4 antibody-guided exclusion diet improves symptoms and rectal compliance in irritable bowel syndrome. Scand J Gastroenterol 2005;40:800-7.

28. Atkinson W, Sheldon TA, Shaath $\mathrm{N}$ et al. Food elimination based on IgG antibodies in irritable bowel syndrome: a randomised controlled trial. Gut 2004;53:1459-64

29. Carroccio A, Scalici C, Di Prima L et al. IgG anti-betalactoglobulin in children with IBS symptoms: a valid aid to decide for the elimination diet. Gut 2005;53:1459-64 (e-letters).

30. Iacono G, Carroccio A, Cavataio F et al. Gastroesophageal reflux and cow's milk allergy in infants: a prospective study. J Allergy Clin Immunol 1996;97:822-7.

31. Ebo DG, Bridts CH, Hagendorens MM et al. Basophil activation test by flow cytometry: present and future applications in allergology. Cytometry B Clin Cytom 2008;74:201-10.

32. Erdmann SM, Heussen N, Moll-Slodowy S et al. CD63 expression on basophils as a tool for the diagnosis of pollen-associated food allergy: sensitivity and specificity. Clin Exp Allergy 2003;33:607-14.

33. Dubois AE, van der Heide S. Basophil-activation tests in Hymenoptera allergy. Curr Opin Allergy Clin Immunol 2007;7:346-9.

34. Leysen J, Sabato V, Verweij MM et al. The basophil activation test in the diagnosis of immediate drug hypersensitivity. Expert Rev Clin Immunol 2011;7:349-55.

35. Ocmant A, Mulier S, Hanssens L et al. Basophil activation tests for the diagnosis of food allergy in children. Clin Exp Allergy 2009;39:1234-45.

36. Ciepiela O, Zwiazek J, Zawadzka-Krajewska A et al. Basophil activation test based on the expression of CD203c in the diagnostics of cow milk allergy in children. Eur J Med Res 2010;15:21-6. 
37. Carroccio A, Brusca I, Mansueto P et al. A cytological assay for diagnosis of food hypersensitivity in patients with irritable bowel syndrome. Clin Gastroenterol Hepatol 2010;8:254-60.

38. Carroccio A, Brusca I, Mansueto P et al. A comparison between two different in vitro basophil activation tests for gluten- and cow's milk protein sensitivity in irritable bowel syndrome (IBS)-like patients. Clin Chem Lab Med 2013;51:1257-63.

39. Bucci C, Zingone F, Russo I et al. Gliadin does not induce mucosal inflammation or basophil activation in patients with non-celiac gluten sensitivity. Clin Gastroenterol Hepatol 2013;11:1294-9.e1.

40. Massari S, Liso M, De Santis L et al. Occurrence of nonceliac gluten sensitivity in patients with allergic disease. Int Arch Allergy Immunol 2011;155:389-94.

41. Branum AM, Lukacs SL. Food allergy among children in the United States. Pediatrics 2009;124:1549-55.

42. Sicherer SH, Furlong TJ, Munoz-Furlong A et al. A voluntary registry for peanut and tree nut allergy: characteristics of the first 5149 registrants. J Allergy Clin Immunol 2001;108:128-32.

43. Skripak JM, Matsui EC, Mudd K et al. The natural history of IgE-mediated cow's milk allergy. J Allergy Clin Immunol 2007;120:1172-7.

44. Sapone A, Lammers K, Casolaro V et al. Divergence of gut permeability and mucosal immune gene expression in two gluten-associated conditions: celiac disease and gluten sensitivity. BMC Med 2011;9:23.

45. Aziz I, Evans KE, Hopper AD et al. A prospective study into the aetiology of lymphocytic duodenosis. Aliment Pharmacol Ther 2010;32:1392-7.

46. Hogan SP, Mishra A, Brandt EB et al. A pathological function for eotaxin and eosinophils in eosinophilic gastrointestinal inflammation. Nat Immunol 2001;2:353-60.

47. Rothenberg ME. Eosinophilic gastrointestinal disorders (EGID). J Allergy Clin Immunol 2004;113:11-28.

48. Ravelli A, Villanacci V, Chiappa S et al. Dietary protein-induced proctocolitis in childhood. Am J Gastroenterol 2008;103:2605-12.

49. Carroccio A, Iacono G, Di Prima L et al. Food hypersensitivity as a cause of rectal bleeding in adults. Clin Gastroenterol Hepatol 2009;7:120-2.

50. Iacono G, Ravelli A, Di Prima L et al. Colonic lymphoid nodular hyperplasia in children: relationship to food hypersensitivity. Clin Gastroenterol Hepatol 2007;5:361-6.
51. Kurppa K, Collin P, Viljamaa M et al. Diagnosing mild enteropathy celiac disease: a randomized, controlled clinical study. Gastroenterology 2009;136:816-23.

52. Kurppa K, Ashorn M, Iltanen S et al. Celiac disease without villous atrophy in children: a prospective study. J Pediatr 2010;157:373-80.

53. Salmi TT, Collin P, Korponay-Szabó IR et al. Endomysial antibody-negative coeliac disease: clinical characteristics and intestinal autoantibody deposits. Gut 2006;55:1746-53.

54. Carroccio A, Iacono G, D’Amico D et al. Production of anti-endomysial antibodies in cultured duodenal mucosa: usefulness in coeliac disease diagnosis. Scand J Gastroenterol 2002;37:32-8.

55. Carroccio A, Iacono G, Di Prima L et al. Antiendomysium antibodies assay in the culture medium of intestinal mucosa: an accurate method for celiac disease diagnosis. Eur J Gastroenterol Hepatol 2011;23:1018-23.

56. Shepherd SJ, Parker FC, Muir JG et al. Dietary triggers of abdominal symptoms in patients with irritable bowel syndrome: randomized placebo-controlled evidence. Clin Gastroenterol Hepatol 2008;6:765-71.

57. Biesiekierski JR, Rosella O, Rose R et al. Quantification of fructans, galacto-oligosacharides and other short-chain carbohydrates in processed grains and cereals. J Hum Nutr Diet 2011;24:154-76.

58. Biesiekierski JR, Peters SL, Newnham ED et al. No effects of gluten in patients with self-reported non-celiac gluten sensitivity following dietary reduction of low-fermentable, poorly-absorbed, short-chain carbohydrates. Gastroenterology 2013;145:320-8.e1-3.

59. Simren M, Barbara G, Flint HJ et al. Intestinal microbiota in functional bowel disorders: a Rome foundation report. Gut 2013;62:159-76.

60. Verdu EF, Huang X, Natividad J et al. Gliadin-dependent neuromuscular and epithelial secretory responses in gluten-sensitive HLA-DQ8 transgenic mice. Am J Physiol Gastrointest Liver Physiol 2008;294:G217-25.

61. Carroccio A, Mansueto P, Morfino G et al. Oligoantigenic diet in the treatment of chronic anal fissures. Evidence for a relationship between food hypersensitivity and anal fissures. Am J Gastroenterol 2013;108: 825-32.

62. Vazquez-Roque MI, Camilleri M, Smyrk T et al. A controlled trial of gluten-free diet in patients with irritable bowel syndrome-diarrhea: effects on bowel frequency and intestinal function. Gastroenterology 2013;144: 903-11. 